\title{
Medición del nivel de satisfacción de vecinos y vecinas con los resultados del Programa Quiero mi Barrio en Chile
}

\author{
Sandra Cortés Chávez ${ }^{1}$, \\ Héctor Sepúlveda Serrano ${ }^{2}$
}

Fecha de recepción: 7 de octubre de 2015

Fecha de aprobación: 7 de marzo de 2016

\begin{abstract}
Resumen
Desde 2006 el Ministerio de Vivienda y Urbanismo de Chile, a través de las Secretarías Regionales de Vivienda y Urbanismo, implementa el Programa Quiero mi Barrio, destinado al mejoramiento urbano y social de barrios vulnerables.

A fines de 2009, en la Región Metropolitana, egresan los primeros 12 barrios intervenidos, surgiendo el interés por medir el nivel de satisfacción de los vecinos y vecinas con los resultados de la intervención, para lo cual se desarrolla una investigación durante el segundo semestre de 2011, que busca definir un modelo de evaluación de satisfacción con los resultados del Programa.

El presente artículo informa de la metodología definida para la medición de la satisfacción y los resultados obtenidos, en el que se manifiesta el alto nivel de satisfacción que el Programa Quiero Mi Barrio alcanza entre los vecinos y vecinas, así como la verificación de que las expectativas eran superiores a los logros alcanzados en la intervención del Programa.

La investigación indagó respecto de la identificación de variables asociadas al nivel de satisfacción. Mediante el análisis de componentes principales aplicado a la evaluación de la caracterización de barrio, se identifican seis factores, cinco de los cuales expresan diferencias significativas con el nivel de satisfacción, a excepción del factor conflictividad.
\end{abstract}

Palabras clave: políticas públicas, nivel de satisfacción de vecinos y vecinas, Programa Quiero Mi Barrio, intervención barrial, sociología urbana.

1 Doctora en Urbanismo, Periferias y Sostenibilidad Urbana, Departamento de Urbanística y Ordenación del Territorio, Universidad Politécnica de Madrid, España. scortesc@minvu.cl; Encargada Estudios y Asistencia Técnica Programa de Recuperación de Barrios, Seremi Minvu Región Metropolitana.

2 Sociólogo, Magíster en Sociología, Universidad de Chile, Santiago, Chile. Contacto: sepulvedaserrano@gmail.com 


\title{
Measuring the satisfaction level in neighbors in relation to the outcomes of Quiero mi Barrio Program in Chile
}

\begin{abstract}
Since 2006, the Chilean Ministry of Housing and Urbanism, through the Regional Secretaries of Housing and Urban Development, implements Quiero mi Barrio (I love my neighborhood) Program, intended for improving vulnerable neighborhoods in urban and social terms.

In late 2009, the first 12 targeted neighborhoods graduated in the Metropolitan Region, which generated interest in measuring the level of satisfaction of residents in relation to the intervention outcomes. To that end, a research took place during the second half of 2011 to define a model for evaluating this level of satisfaction.

This article informs about the methodology defined for measuring satisfaction and outcomes and the high level of satisfaction that this Program generated in neighbors. It also verifies that expectations were higher than the achievements of the program intervention.

The research explored the identification of variables associated with satisfaction. An analysis of the main components applied to the evaluation of a neighborhood characterization identified six factors and five of them expressed significant differences in the level of satisfaction, except for the factor related to conflict.
\end{abstract}

Keywords: public policies, neighbors' level of satisfaction, Quiero Mi Barrio Program, neighborhood intervention, urban sociology

\section{Medição do nível de satisfação de vizinhos com os resultados do Programa Quero meu Bairro no Chile}

\footnotetext{
Resumo

Desde 2006, o Ministério de Vivenda e Urbanismo do Chile, através das Secretarias Regionais de Habitação e Urbanismo implementa o programa Quero o meu Bairro, destinado ao melhoramento urbano e social dos bairros vulneráveis.

No final de 2009, na Região Metropolitana, graduam-se os primeiros 12 bairros intervindos, surgindo o interesse em medir o nível de satisfação dos vizinhos com os resultados da intervenção, para os quais se desenvolve uma investigação durante a segunda metade do ano 2011, que procura definir um modelo da avaliação de satisfação com os resultados do programa.

Este artigo informa da metodologia definida para medir a satisfação e os resultados obtidos, no qual se manifesta o alto nível de satisfação que o programa Quero meu Bairro alcança entre os vizinhos, assim como a verificação de que as expectativas foram superiores aos logros realizados pela intervenção do programa.

A pesquisa investigou a respeito da identificação de variáveis associadas ao nível de satisfação. Através da análise de componentes principais aplicado à
} 
avaliação de caracterização do bairro, identificam-se seis fatores, dos quais cinco expressam diferenças significativas com o nível de satisfação, com exceção do fator conflito.

Palavras-chave: políticas públicas, nível de satisfação de vizinhos, Programa Quero meu Bairro, a intervenção do bairro, sociologia urbana.

\section{Introducción}

El Programa Quiero Mi Barrio (PQMB) está inserto y forma parte de una política pública que se implementa a escala barrial desde 2006 por el Ministerio de Vivienda y Urbanismo (MINVU) de Chile, en asociación con municipios y actores de la comunidad de barrios vulnerables que son objeto de la intervención.

Tiene por finalidad: "Contribuir al mejoramiento de la calidad de vida de los habitantes de barrios que presentan deterioro urbano, vulnerabilidad social, a través de un proceso participativo de recuperación de espacios públicos y de los entornos urbanos de familias" (MINVU, 2008, p. 6), en el cual los habitantes del barrio participan del diagnóstico de su realidad, en la formulación de propuestas y proyectos, en el diseño e implementación y en la evaluación. Las acciones mencionadas se realizan en un periodo de tres años.

La implementación se aborda mediante el levantamiento de un diagnóstico participativo, la formulación de Plan Maestro de Recuperación Barrial, que contempla intervenciones sociales y urbanas, parte de las cuales se ejecutan mediante un Contrato de Barrio que se define de forma tripartita entre Comunidad ${ }^{3}$, Municipio, y MINVU, todo ello durante la Fase I. Las iniciativas de ejecución social se plasman en un Plan de Gestión Social que aborda temáticas de asociatividad, participación, seguridad, medioambiente e identidad; y la intervención urbana se desarrolla mediante un Plan de Gestión de Obras, centrada en la recuperación y/o habilitación de espacios públicos y equipamientos del barrio, planes que son ejecutados en la Fase II del PQMB.

Representada por una organización que se forma al alero del Programa, denominada Consejo Vecinal de Desarrollo. 
Finalmente, se efectúa el cierre de la intervención con un proceso de sistematización y evaluación participativa que se desarrolla en la Fase III.

Desde 2006 a 2011, en la Región Metropolitana, la Secretaría Regional Ministerial (Seremi) de Vivienda y Urbanismo intervino, a través del Programa Quiero Mi Barrio, más de 80 barrios de distintas comunas, de los cuales 12 concluyeron su intervención a fines de 2009. Si bien se efectuaron mediciones nacionales respecto de la variación de algunos indicadores de la "Encuesta de Percepción, Satisfacción y Caracterización" entre el momento diagnóstico -denominado Fase I- y el momento de término de la intervención -denominado Fase III-, estas responden a la comparación de variables simples medidas mientras el PQMB se encontraba en su fase de ejecución.

En la Región Metropolitana era necesario indagar sobre el nivel de satisfacción de los vecinos y vecinas con el PQMB, en poblaciones donde la intervención había concluido el año 2009, a objeto de comprender el nivel de valoración que atribuyen a los resultados del PQMB.

La investigación se efectuó el año 2011, a dos años de concluido el PQMB; por tanto, correspondió a una medición expost, en la cual se parte de la hipótesis de la existencia de variables de carácter cognitivo y otras asociadas a la significación, capaces de recoger la valoración de la intervención del $\mathrm{PQMB}$ por parte de los vecinos y vecinas en su hábitat. Para su desarrollo se utilizaron los campos teóricos de la satisfacción residencial y la satisfacción del cliente.

\section{Revisión bibliográfica}

\section{La satisfacción del cliente}

En la literatura de la satisfacción al consumidor son varias la investigaciones (Oliver, 1989; Feijoo, Caro, Gil, y Quintana, 2002) que coinciden en que el concepto de "satisfacción" tiene un am- 
plio espectro de definiciones de gran complejidad y difícil aproximación, ya que no existe consenso ante un contexto determinado y, por lo tanto, la definición de estándares o parámetros es compleja. Por ello, las investigaciones en este campo parten de la explicación de las variables que inciden en la satisfacción mediante la ejecución y análisis de modelos, más que en la definición del concepto en sí mismo.

Liljander y Strandvik (1997, citados en Femenía, s. f.) comprenden la satisfacción como una respuesta del consumidor, consecuencia de la evaluación resultado del producto y un estándar de comparación. Desde otro enfoque, Kotler (1998, en Gelvez, 2010) plantea que la satisfacción es un sentimiento resultante de la evaluación del desempeño o resultado del producto o servicio en relación con las expectativas.

Sin embargo, existe coincidencia entre diversos investigadores respecto de que la medición de la satisfacción está vinculada al consumo de productos o de servicios, donde los resultados entregan información relevante para mejorar la calidad de los productos ofertados, o, en el ámbito de políticas públicas, mejorar la calidad de los servicios.

Feijoo et al. (2002) revisan distintos modelos para explicar el comportamiento de la satisfacción del consumidor, distinguiendo dos enfoques. El primero, de la satisfacción como resultado de un uso, servicio o de un producto, donde el enfoque es principalmente economicista. El segundo, un enfoque psicológico centrado en la satisfacción como proceso evaluativo, en el cual lo cognitivo entra como dimensión al comparar rendimiento con expectativas, y como proceso afectivo en el que entran las emociones que el consumo genera. De estos dos enfoques se derivan modelos de medición de la satisfacción.

Desde el enfoque cognitivo se plantea que el consumidor analiza y evalúa el servicio, resultado o producto desde una perspectiva racional entre resultado del producto o servicio y un estándar definido de comparación (Liljander y Strandvik, 
1997, citado en Femenía, s. f.). Dentro de este enfoque se encuentran:

- Modelo de confirmación de expectativas: en el cual se entiende la satisfacción producto de la comparación de la realidad percibida con algún tipo de estándar. La satisfacción se explica en relación a las expectativas y el rendimiento, identificándose tres resultantes: a) expectativa confirmada, cuando el producto es lo esperado; b) disconfirmación positiva, cuando el producto supera la expectativa, o c) disconfirmación de expectativa, cuando el producto es inferior a lo esperado (Oliver, 1989).

- Basado en la teoría de la equidad: plantea que la equidad y la injusticia inciden en el nivel de valoración de la satisfacción. La equidad como estándar de comparación del costo y beneficio; y la discrepancia o inequidad como estándar de expectativa o rendimiento. El individuo considera a terceros que forman parte de la transacción en la evaluación de costo beneficio (Huppertz, 1978, citado en Feijoo et al., 2002).

- Modelo de discrepancia del valor percibido: la satisfacción es un efecto perceptual de la evaluación, en el que la comparación se hace en relación a los principios o valores del individuo. A menor diferencia entre la percepción de evaluación y los valores del consumidor, mayor es la satisfacción (Westbrook y Reilly, 1983, citado en Femenía et al., s. f.).

- Basado en la teoría de atribución causal: considera que la satisfacción del individuo es influenciada por causas del éxito o fracaso en la experiencia de consumo de un producto o servicio, distinguiendo aquellas atribuciones debidas al propio cliente y aquellas que son causa del ambiente o entorno (Martínez-Tur, Peiró y Ramos, 2001, citado en Feijoo et al., 2002).

- Modelo de nivel de comparación: la satisfacción es entendida como respuesta del consumidor a la diferencia entre resultado del servicio y nivel de comparación, donde este último es influenciado por: a) experiencia similar con otros productos; 
b) referente de experiencia de otros consumidores, y c) promesas del proveedor (Latour y Peat, 1970, citado en Femenía, s. f.).

Otro enfoque de evaluación de satisfacción lo constituyen los modelos afectivos cognitivos, los cuales atribuyen la satisfacción tanto a la evaluación de la utilidad del producto o servicio con estándares, como a las emociones que la experiencia de consumo genera como parte de un proceso; uno de ellos es:

- Modelo afectivo cognitivo (Oliver, 1981, 1989, citado en Femenía, s. f.): parte de la base de los modelos cognitivos en los que se evalúa la experiencia de éxito o fracaso del consumo y como consecuencia una emoción (agrado o desagrado del consumidor), para en un segundo momento manifestar una reacción de emociones que deriva en una acción (Zeithaml, 1988, citado en Femenía, s. f.). Al explicar las variables de la satisfacción, distingue que en el ámbito de los servicios y productos no duraderos prima lo afectivo; mientras que en productos duraderos lo cognitivo prima en la evaluación.

- Modelo cognitivo afectivo de los servicios de turismo (Andreu y Bigné, 2004): indican que la disconfirmación (medición del resultado obtenido en el servicio en comparación con las expectativas) genera en el campo de las emociones un estado de agrado o desagrado, junto con la activación o desactivación del individuo. Ambas variables -agrado y accióngeneran la satisfacción y desencadenan a su vez intenciones de comportamiento con efectos mediatos, como la lealtad al servicio y la disposición a pagar por el servicio; y efectos inmediatos, como la intensificación del uso del producto. Se establece la relación causal de los resultados de la comparación entre parámetros y expectativas: a mayor número de expectativas cumplidas o superadas por el servicio, mayor agrado; a mayor agrado, mayor activación y, como consecuencia, mayor satisfacción. A su vez, a mayor satisfacción, mayores intenciones de comportamiento positivo. 
Los modelos afectivos cognitivos parecen ser los más idóneos para medir la satisfacción en el barrio, ya que, atendiendo a las variables intervenidas por PQMB luego de un proceso de casi tres años de trabajo continuo, mejora la calidad de espacios públicos y equipamientos, así como las relaciones sociales entre los habitantes del barrio, abordando por tanto la evaluación del servicio prestado al habitante como consumidor de la oferta pública. Abordaría a lo menos dos dimensiones: aquellas de naturaleza tangible, como también elementos intangibles que se manifiestan en las interacciones de los consumidores o habitantes en su hábitat.

\section{La satisfacción residencial}

Vistos los enfoques de los modelos de satisfacción del cliente, indagamos respecto de la satisfacción residencial, a objeto de identificar las variables que se consideran en estudios de esta naturaleza, atendiendo que se trata explorar la satisfacción con los resultados de un PQMB que interviene en el barrio.

En la satisfacción residencial nos encontramos nuevamente con diversas variables de composición del concepto, donde emergen aspectos provenientes de las características físicas y experiencias de carácter afectivo, cognitivo y conductual que inciden en la satisfacción del entorno habitado; evaluación que varía en función de las características del individuo, sus experiencias y percepciones, y que nos permitirían afirmar que la satisfacción a escala barrial debería estar relacionada con modelos afectivos cognitivos.

Aragonés y Amérigo (1987, citado en Breymann, 2015) distinguen una serie de variables que inciden en la satisfacción residencial de carácter objetivo, como la funcionalidad, las condiciones y servicios del espacio físico; variables sociales relacionadas con las interacciones, variables perceptuales y otras vinculadas a las características de arraigo del individuo, que generan un efecto en el habitante. Este efecto o sensación de agrado / desagrado 
con el lugar de residencia tiene como referentes parámetros de comparación a otros lugares de la ciudad que permiten establecer diferencias y similitudes, así como imaginarios deseados (Valera, Guardia, Cruells, Paricio, Pol, Reixach, Schilman y Vallés, 1998, citados en Breymann, 2015).

Los planteamientos de Proshanky, Fabian y Kaminott (1983, citados en Breymann, 2015) nos refieren a la relevancia de la satisfacción residencial, la percepción positiva de las características del entorno y la influencia que poseen las variables vinculadas a las interacciones sociales, los logros personales y grupales que suceden en el lugar donde se habita, en particular en cómo éste es un medio para satisfacer necesidades cotidianas. Ello es coincidente con las investigaciones de Adriaase (2007, citado en Breymann, 2015), que sostiene que las características del clima social son uno de los aspectos más significativos en la satisfacción residencial.

A su vez Adriaase (2007, citado en Breymann, 2015) plantea que la satisfacción residencial positiva condiciona el comportamiento de los individuos en su entorno de forma favorable, funcionando como predictor de comportamiento; por tanto, coincidente con el modelo cognitivo afectivo de los servicios de turismo (Andreu y Bigné, 2004).

Desde la satisfacción residencial podemos distinguir varios elementos claves para aproximarnos a la evaluación de intervenciones en un contexto barrial:

- La relevancia de las características de ciertas áreas de la ciudad que actúan en los individuos como referentes de imaginario deseado, en el entendido de que presentan características multidimensionales que favorecen el desarrollo de la vida cotidiana.

- Existencia de variables de naturaleza multidimensional (espaciales, perceptuales, afectivas, cognitivas) que incidirían en la satisfacción residencial. 
- La variabilidad que presentaría la satisfacción residencial en relación a las características del habitante, sus experiencias vividas y percepciones del hábitat.

- La existencia de un vínculo con el lugar, generado por el arraigo e identidad, que tiene relación causal con niveles de agrado o desagrado con el lugar de residencia.

- La relevancia que algunos investigadores asignan a las experiencias, relaciones e interacciones sociales, vinculada a la valoración positiva de la satisfacción residencial.

- La existencia de relaciones entre la satisfacción residencial positiva y la activación favorable con el entorno y su utilización como predictor de comportamiento.

\section{Medición de satisfacción en las poblaciones intervenidas}

Tanto desde los modelos de evaluación de satisfacción del cliente, como desde la satisfacción residencial, nos encontramos con que el concepto de "satisfacción" respondería a un constructo cuyas dimensiones permitirían al investigador efectuar su medición.

La satisfacción estaría relacionada con un marco o parámetro de referencia externo, que daría cuenta de la expectativa y permite efectuar el proceso de evaluación, en el cual el individuo se categoriza como "usuario" de un "producto o servicio" que se somete a evaluación, dando cuenta de una operación matemática que tiene como efecto una emoción que condiciona una acción posterior.

Intrínsecamente, este proceso evaluativo significa un distanciamiento con el producto o servicio que permite observar y efectuar su acto evaluativo. En el nivel de habitante, este proceso también implica una abstracción de la realidad cotidiana, un distanciamiento para efectuar el proceso evaluativo, quedando el habitante como espectador de una realidad experimentada en el hábitat.

Ambos procesos evaluativos de productos o servicios y del hábitat presentan una diferencia significativa respecto de cómo 
el usuario se relaciona con el objeto de evaluación (producto, servicio y entorno residencial). Para productos y servicios se parte del interés del usuario por adquirir el producto o ser usuario del servicio que presta una empresa o una institución, y, por tanto, supone un proceso de elección del individuo que se moviliza por el interés. Es el interesado el que acude a comprar o a utilizar el servicio.

En el lugar de residencia la evaluación es distinta. En los contextos en los cuales efectuamos el estudio, los habitantes, por su condición, no tuvieron la posibilidad de elegir, entre un amplio espectro de poblaciones, donde residir; en su mayoría son asignatarios de subsidios estatales, lo cual restringió las opciones de elegibilidad, una primera condición -relevante a nuestro entender- en la concepción del modelo de evaluación.

Una segunda cuestión dice relación con la condición de elegibilidad o la solicitud de los residentes para que el Programa Quiero Mi Barrio se desarrollara en su población. Las poblaciones y comunidades intervenidas no fueron partícipes de decidir o convocar al PQMB para que este llegara a sus comunidades, fueron los municipios y el gobierno regional quienes decidieron aquello.

Es necesario considerar que, en este caso en particular, los usuarios no tuvieron la opción de elegir el servicio y productos entregados con la intervención del PQMB, aunque el desarrollo en sí mismo del Programa es participativo.

La intervención de estas poblaciones es interés del municipio y del gobierno regional por mejorar la calidad de vida de los habitantes de poblaciones vulnerables, partiendo del supuesto de que los habitantes se interesan por las materias que el PQMB plantea en su desarrollo.

En esta línea de trabajo, Forero y Ospina (2013) indican que los productos provocan experiencias de agrado y mayor satisfacción cuando dan cobertura a las necesidades que son de interés del individuo. Asimismo, Santomá (2008, citado en Gelvez, 2010) 
indica que la opinión del cliente es lo más importante a considerar, lo que se manifiesta por sus necesidades y expectativas.

A nuestro entender, este planteamiento es clave para comprender que la construcción del modelo de evaluación de la satisfacción con los resultados del PQMB debe abordar la evaluación del resultado junto con el grado de importancia o significación que éste atribuye a aquella intervención, ya que aquello permitirá clarificar si las materias desarrolladas por el PQMB en el barrio son de interés y motivación de los vecinos, para desde estas dos variables evaluar los resultados de la satisfacción.

\section{Evaluación de la satisfacción en la calidad de los servicios}

Se indagó respecto de modelos de satisfacción que utilicen la variable "importancia del producto o servicio desde la perspectiva del usuario y desde la evaluación del resultado o desempeño del producto", encontrando referentes de modelos de satisfacción de clientes con el servicio:

- Modelo SERVQUAL: Zeithaml, Parasuraman y Berry (1998, citados en Gelvez, 2010) plantean que la medición de la calidad del servicio se efectúa mediante la diferencia entre expectativa y desempeño, medida a través del GAP, o diferencia entre expectativa y percepción. El modelo se construye con cinco dimensiones: elementos tangibles, fiabilidad, capacidad de respuesta, seguridad y empatía.

- Modelo SERVPERF: Cronin y Taylor (1992, citados en Gelvez, 2010) definen que la calidad del servicio es una actitud del consumidor, formada a través de la percepción, siendo las escalas de medición ajustadas para cada caso.

- Modelo de análisis importancia-desempeño IPA (ImportancePerformance Análysis): Kitcharoen (2004, citado en Gelvez, 2010) identifica fortalezas y debilidades de la importancia relativa de la oferta y la evaluación de la oferta, permitiendo 
efectuar un análisis entre la satisfacción y la importancia de los productos para los clientes, donde los valores de la importancia-como del desempeño de la oferta o atributos-conforman un cuadrante, indicado en la figura 1. Blanco (2007) representa el modelo en cuadrantes, que muestran diversas combinaciones de importancia y desempeño de los atributos o productos, de utilidad para definir estrategias y priorizar mejoras en función de las fortalezas y debilidades encontradas en la matriz.

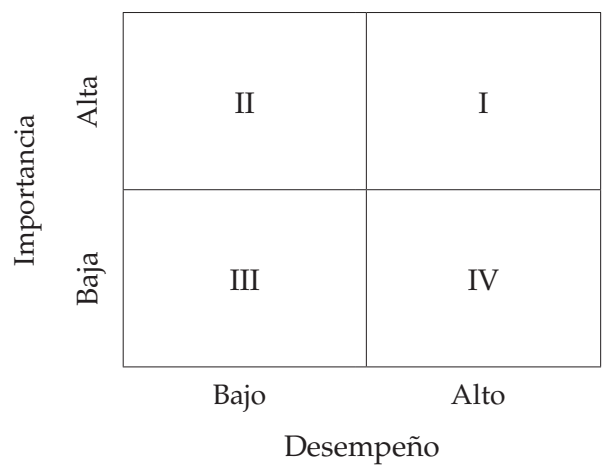

Figura 1: Fuente: Blanco (2007).

La escala importancia-desempeño, de acuerdo con los planteamientos de Mullins y Spetich (1998, citados en Gelvez, 2010), se basa en que la satisfacción se afecta por la importancia de un atributo, así como por el rendimiento percibido del mismo.

\section{Metodología}

La investigación es de naturaleza descriptiva, utilizando para su desarrollo investigación cuantitativa. Se implementa para la evaluación de la intervención de 12 barrios, aplicando un cuestionario para medir el nivel de satisfacción de los vecinos y vecinas del barrio, y para conocer qué variables pudieran estar relacionadas o asociadas al nivel de satisfacción.

Para el diseño de la evaluación se utiliza la adaptación del Modelo Importance-Performance Análysis, siendo definida la 
satisfacción como el grado de valoración de los proyectos contenidos en la propuesta de intervención del PQMB y los resultados obtenidos con la ejecución de ellos en cada barrio.

Operativamente, el nivel de satisfacción permitiría conocer si, para los vecinos y vecinas, la ejecución del PQMB en el barrio respondió a la expectativa que tenían con la intervención, y la evaluación que ellos y ellas realizan de cada uno de los proyectos ejecutados en el barrio, que fueron parte de lo que se denominó Plan Maestro de Recuperación Barrial.

\section{Construcción del Índice de Satisfacción}

Para medir el nivel de satisfacción se construyó un Índice de Satisfacción de los vecinos y vecinas del barrio, con base en dos dimensiones: a) importancia otorgada, por los vecinos y vecinas, a los proyectos que se incluyeron en el Contrato de Barrio ${ }^{4}, \mathrm{y}$ b) evaluación de los vecinos y vecinas de los proyectos ejecutados en su barrio.

\section{Matriz para análisis del nivel de satisfacción}

Para el análisis de los resultados se construyó una matriz bidimensional, adaptando la Matriz de Desempeño de Blanco (2007) y relacionando el nivel de importancia de los proyectos que se consideraron ejecutar en el barrio y la evaluación que los vecinos y vecinas realizan de los proyectos ejecutados por del PQMB en el barrio. El eje horizontal corresponde a la importancia y el eje vertical representa la evaluación. Esta gráfica permite obtener una perspectiva de la satisfacción que los vecinos declaran con los resultados alcanzados por la acción del PQMB.

\footnotetext{
$4 \quad$ Instrumento de acuerdo entre la comunidad, el Municipio y el MINVU, en el que se comprometen una serie de obras e iniciativas sociales a desarrollar con financiamiento del Programa Quiero Mi Barrio.
} 
El gráfico de dispersión que resulta de esta relación da cuenta del nivel de satisfacción. Figura 2.

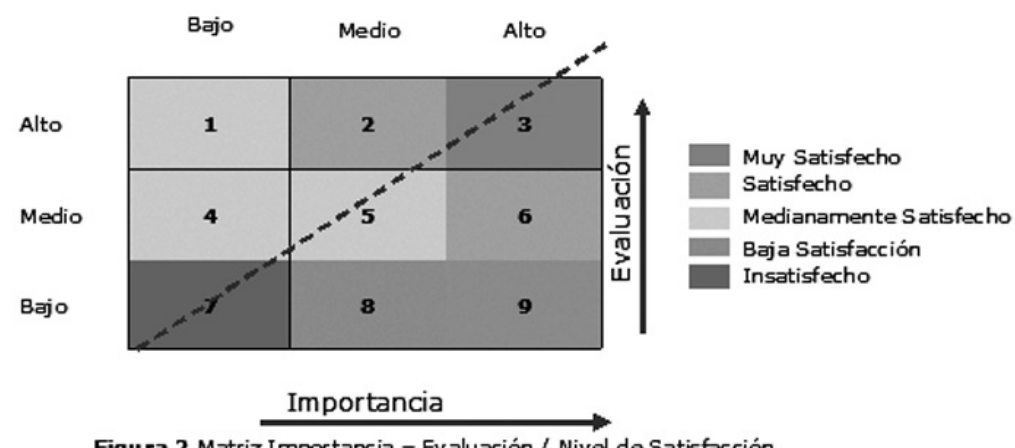

Figura 2 Matriz Importancia-Evaluación / Nivel de Satisfacción.

Los casos se posicionan conformando la diagonal: significa que la valoración de la importancia y la evaluación de los resultados se comportan de forma equivalente.

Los casos que se posicionan de la diagonal hacia arriba informan que la evaluación supera a la importancia.

Los casos que se posicionen de la diagonal hacia abajo dan cuenta de que la importancia es mayor que la evaluación.

La lectura de la matriz de importancia-evaluación contempla la estructuración de tres niveles de importancia: bajo, medio, y alto; igual cosa para el nivel de evaluación. Lo que configuró una matriz de nueve celdas que expresan diferentes niveles de satisfacción, cuyo significado se expresa de la siguiente manera:

1. INSATISFECHO, corresponde al cuadrante 7.

2. BAJA SATISFACCIÓN, los cuadrantes 8 y 9.

3. MEDIANA SATISFACCIÓN, los cuadrantes 1,4 y 5 .

4. SATISFACCIÓN, los cuadrantes 2 y 6 .

5. ALTA SATISFACCIÓN, el cuadrante 3. 


\section{Análisis factorial}

Para la discusión de los resultados, se procedió a indagar respecto de variables asociadas a nivel de satisfacción, para lo cual se procesaron los ítemes referidos a la caracterización del barrio a través de análisis factorial de componentes principales, y los componentes o factores extraídos se sometieron a la prueba estadística de $\mathrm{t}$ Student, con un $\mathrm{P}=<0,05$ para diferencia de medias.

\section{Tamaño muestral}

El marco muestral corresponde a 12 barrios egresados durante el año 2009 del PQMB, según la siguiente tabla N¹:

Tabla $\mathrm{N}^{\circ}$ 1: Barrios Egresados el 2009 y $\mathrm{N}^{\circ}$ de viviendas.

\begin{tabular}{|c|c|c|c|c|}
\hline COMUNA & BARRIO & $\begin{array}{c}\mathrm{N}^{\circ} \text { de } \\
\text { viviendas }\end{array}$ & Muestra & $\begin{array}{l}\mathrm{N}^{\circ} \text { de en- } \\
\text { cuestados }\end{array}$ \\
\hline 1 Cerro Navia & Población Santa Clara & 583 & 120 & 81 \\
\hline 2 El Bosque & $\begin{array}{l}21 \text { de Marzo - Héroes del } \\
\text { Morro }\end{array}$ & 318 & 120 & 83 \\
\hline 3 El Bosque & Santa Elena Sub. Sector 2 & 340 & 120 & 107 \\
\hline 4 El Bosque & Villa San Francisco I y II & 258 & 120 & 79 \\
\hline 5 La Granja & $\begin{array}{l}\text { Población San Gregorio } \\
\text { Sector } 1\end{array}$ & \multirow{2}{*}{1.030} & \multirow{2}{*}{120} & \multirow{2}{*}{103} \\
\hline 6 La Granja & $\begin{array}{l}\text { Población San Gregorio } \\
\text { Sector } 2\end{array}$ & & & \\
\hline 7 La Pintana & 21 de Mayo Sector 2 & 485 & 120 & 111 \\
\hline 8 Peñalolén & La Alborada & 418 & 120 & 76 \\
\hline 9 Recoleta & Marta Colvin & 136 & 136 & 55 \\
\hline 10 Recoleta & Santa Ana & 309 & 120 & 97 \\
\hline 11 San Ramón & Modelo Sector 1 & \multirow{2}{*}{426} & \multirow{2}{*}{120} & \multirow{2}{*}{91} \\
\hline 12 San Ramón & Modelo Sector 2 & & & \\
\hline & & 4.303 & 1.236. & 883 \\
\hline
\end{tabular}

Se definió encuestar una persona por vivienda, con un máximo de 120 vecinos y vecinas por barrio, excepto el barrio Marta Colvin, en el que se tomó la decisión de encuestar a 136. Para la selec- 
ción debían cumplir dos condiciones: tener 18 años o más, y vivir en el barrio a lo menos desde el año 2006.

Se configuró una muestra no probabilística de vecinos y vecinas de los 12 barrios egresados del PQMB en el transcurso del año 2009, de tamaño $n=1.236$. La muestra efectiva fue $n=883$, que representa un $71 \%$ del tamaño muestral inicialmente definido.

\section{El instrumento de medición}

Se diseñó un cuestionario con 47 preguntas cerradas, que median tres dimensiones a observar:

La primera dimensión corresponde a la opinión que los vecinos tienen del barrio.

a) Características del barrio.

b) Organizaciones sociales.

c) Preocupación por espacio público.

d) Relación con vecinos y vecinas.

e) Participación.

f) Identidad.

g) Seguridad.

La segunda dimensión corresponde a la Evaluación Nivel de Satisfacción:

a) Plan de Gestión de Obras, donde se evalúan 95 obras, clasificadas en 10 tipologías: telecomunicaciones; obras complementarias; mobiliario; equipamiento; circulación; áreas verdes; arbolado; alumbrado y aguas lluvias.

b) Acciones del PQMB en el espacio público, incorporó las acciones en el área del Plan de Gestión Social del Programa.

c) Evaluación de la Gestión en el Espacio Social.

La tercera dimensión corresponde a la Evaluación del Plan de Gestión Social:

a) Organización barrial Consejo Vecinal de Desarrollo.

b) Organización medioambiental. 
c) Historia del barrio.

d) Actores sociales.

e) Estrategia comunicacional.

\subsection{Pre test}

El cuestionario fue testeado en el barrio 6 de Mayo de La Pintana y barrio Modelo de San Ramón, lo que permitió revisar y corregir la comprensión de las preguntas, alternativas de respuesta y escalas de evaluación, revisar la codificación y las instrucciones del cuestionario e identificar requerimientos de la salida a terreno. El testeo además permitió medir el nivel de confiabilidad de las diversas escalas con las cuales se construyó el Índice de Satisfacción.

\subsection{Confiabilidad}

Se efectuó un test de fiabilidad del cuestionario para comprobar la entrega de resultados consistentes para cada una de sus dimensiones:

I. Se construyó una escala para evaluar la percepción del barrio que tienen los vecinos y vecinas. El análisis de confiabilidad de esta escala arrojó un Alpha = ,7899.

II. El Índice de Satisfacción se construyó con tres escalas:

a) Importancia y evaluación con la ejecución de las obras del barrio.

b) Importancia y evaluación con la gestión en el espacio público.

c) Importancia y evaluación con la gestión o acción del PQMB en el espacio social.

El análisis de confiabilidad para estas tres escalas arrojó un Alpha $=, 7081$.

III. También se construyó una escala para medir el nivel de conocimiento e información de los vecinos y vecinas respecto del Programa Quiero mi Barrio.

El análisis de confiabilidad arrojó un Alpha = ,7909. 


\section{Resultados}

\section{Caracterización de encuestados}

Del total de encuestas aplicadas y válidas, la mayoría de los entrevistados son mujeres. La tabla $\mathrm{N}^{\circ} 2$ muestra el número de cuestionarios aplicados por barrio según sexo.

Predomina un 38\% de mujeres de rango etario entre 40 a 59 años, mientras que un $47 \%$ de los hombres encuestados es del rango etario 60 y más años de edad. Los encuestados correspondieron mayoritariamente a jefes de hogar (53\%); de ellos, las mujeres representan un $45 \%$, y los hombres un $74,2 \%$.

Tabla $N^{\circ}$ 2: Total encuestas válidas por barrio, por sexo del encuestado.

\begin{tabular}{|c|c|c|c|c|c|c|c|}
\hline & & \multicolumn{4}{|c|}{ Sexo encuestado(a) } & \multicolumn{2}{|c|}{ Total } \\
\hline & & \multicolumn{2}{|c|}{ Hombre } & \multicolumn{2}{|c|}{ Mujer } & \multirow[b]{2}{*}{ Recuento } & \multirow[b]{2}{*}{$\%$ fila } \\
\hline & & Recuento & $\%$ fila & Recuento & $\%$ fila & & \\
\hline \multirow[t]{10}{*}{ Barrios } & 6 de Mayo & 30 & $27,0 \%$ & 81 & $73,0 \%$ & 111 & $100,0 \%$ \\
\hline & San Gregorio & 23 & $23,7 \%$ & 74 & $76,3 \%$ & 97 & $100,0 \%$ \\
\hline & Población Modelo & 40 & $40,0 \%$ & 6 & $60,0 \%$ & 100 & $100,0 \%$ \\
\hline & Villa San Francisco de Asis & 14 & $17,9 \%$ & 64 & $82,1 \%$ & 78 & $100,0 \%$ \\
\hline & 21 de Marzo - Héroes del Morro & 15 & $18,5 \%$ & $\infty 6$ & $81,5 \%$ & 81 & $100,0 \%$ \\
\hline & Santa Elena & 28 & $26,9 \%$ & 76 & $73,1 \%$ & 104 & $100,0 \%$ \\
\hline & La Alborada & 21 & $28,0 \%$ & 54 & $72,0 \%$ & 75 & $100,0 \%$ \\
\hline & Marta Colvin & 12 & $22,2 \%$ & 42 & $77,8 \%$ & 54 & $100,0 \%$ \\
\hline & Santa Ana & 28 & $28,9 \%$ & $\oplus$ & $71,1 \%$ & 97 & $100,0 \%$ \\
\hline & Santa Clara & 29 & $35,8 \%$ & 52 & $64,2 \%$ & 81 & $100,0 \%$ \\
\hline Total & & 240 & $27,3 \%$ & 638 & $72,7 \%$ & 878 & $100,0 \%$ \\
\hline
\end{tabular}

Respecto de los años de antigüedad en el barrio de los encuestados. La tabla $\mathrm{N}^{\circ} 3$ informa la distribución, desagregada por sexo, considerando año de corte 1980.

Tabla N 3: Año en que llegó a vivir al barrio según sexo.

\begin{tabular}{|c|c|c|c|c|c|c|c|}
\hline & \multicolumn{4}{|c|}{ Sexo encuestado(a) } & \multicolumn{2}{|c|}{ Total } \\
\hline & & \multicolumn{2}{|c|}{ Hombre } & \multicolumn{2}{|c|}{ Mujer } & \multirow[b]{2}{*}{ Recuento } & \multirow[b]{2}{*}{$\%$ col. } \\
\hline & & Recuento & $\%$ col. & Recuento & $\%$ col. & & \\
\hline \multirow{3}{*}{$\begin{array}{l}\text { Años que vive } \\
\text { en el barrio } \\
\text { Total }\end{array}$} & De 1935 a 1980 & 137 & $57,1 \%$ & 294 & $46,1 \%$ & 431 & $49,1 \%$ \\
\hline & De 1981 adelante & 103 & $42,9 \%$ & 344 & $53,9 \%$ & 447 & $50,9 \%$ \\
\hline & & 240 & $100,0 \%$ & 638 & $100,0 \%$ & 878 & $100,0 \%$ \\
\hline
\end{tabular}

\section{Nivel de satisfacción general}

El nivel de satisfacción consolidado de los 12 barrios da cuenta del promedio general, considerando las tres dimensiones consultadas: Plan de Gestión de Obras, Gestión del Espacio Público y 
Gestión Social. Los resultados indican que un 68,7\% de los encuestados se declara muy satisfecho con los resultados del PQMB.

Figura 3: Matriz importancia - evaluación / resultados nivel de satisfacción general 12 barrios.

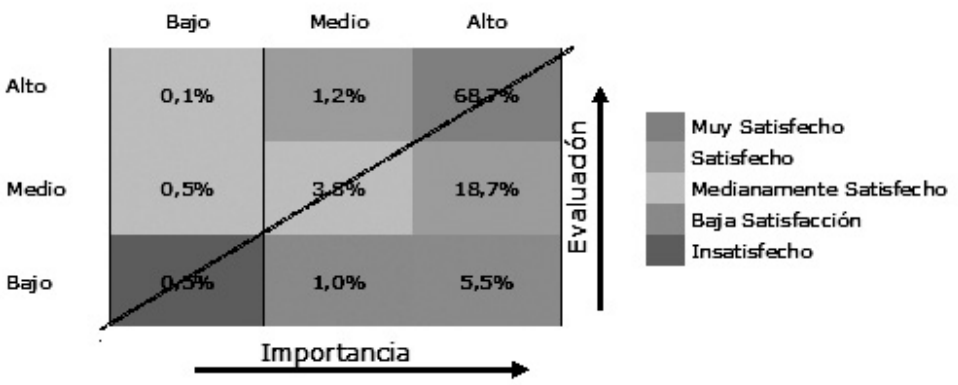

Figura 3: Matriz Importancia-Evaluación/Resultados Nivel de Satisfacción General 12 barrios

La figura $\mathrm{N}^{\circ} 3$ muestra la distribución del nivel de satisfacción de los 12 barrios, en la que la mayoría de los casos se distribuyen concentrados bajo la diagonal, dando cuenta de que la importancia tiene mayor valoración que la evaluación.

Figura $\mathrm{N}^{\circ}$ 4: Nivel de satisfacción promediado de tres dimensiones, 12 barrios.

Promedio General Nivelde satisfación con intervención del PRB por Barrio

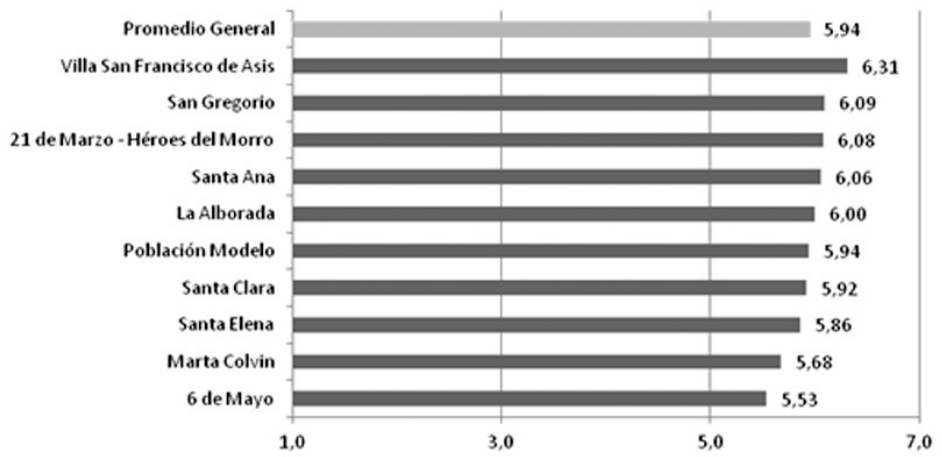

La figura $\mathrm{N}^{\circ} 4$ muestra que el promedio general del nivel de satisfacción de los 12 barrios es 5,9, siendo San Francisco de Asís 
de El Bosque el barrio que tiene más alta satisfacción y 6 de Mayo de La Pintana el barrio con menor nivel de satisfacción

\section{Nivel de satisfacción Plan de Gestión de Obras}

La figura $\mathrm{N}^{\circ} 5$ muestra el gráfico de dispersión. Informa de la distribución del nivel de importancia y evaluación de la ejecución de las obras por cada uno de los encuestados y encuestadas. Se aprecia que un gran volumen de los encuestados tiene un alto nivel de satisfacción. La mayoría de los casos se distribuyen concentrados bajo la diagonal, dando cuenta de que la importancia de las obras tiene mayor valoración que la evaluación.

Figura $N^{\circ}$ 5: Matriz importancia-evaluación / resultados nivel de satisfacción Plan de Gestión de Obras.

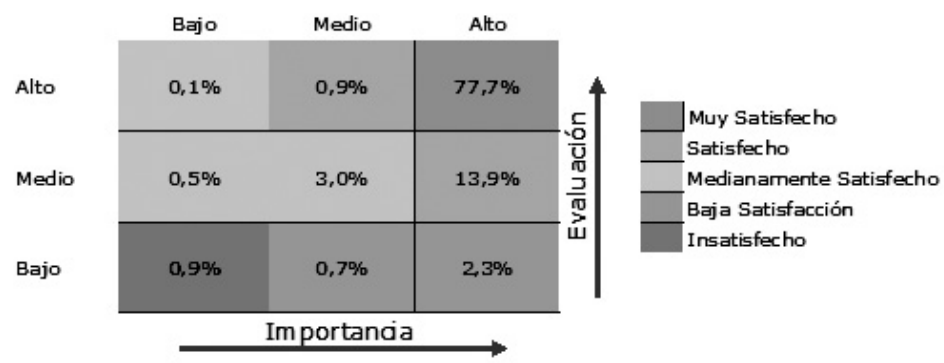

Figura $N^{\circ}$ 5: Matriz Importancia-Evaluación/Resultados Nivel de Satifacción PG O

\section{Nivel de satisfacción Gestión del Espacio Público}

Los resultados del nivel de importancia y de evaluación de la Gestión del Espacio Público dan cuenta de que el nivel de importancia de las acciones en el espacio público tienen mayor relevancia que los resultados finales alcanzados por el aporte del PQMB. Figura $N^{\circ} 6$. 
Figura $\mathrm{N}^{\circ}$ 6: Matriz importancia-evaluación / resultados nivel de satisfacción Gestión del Espacio Público.

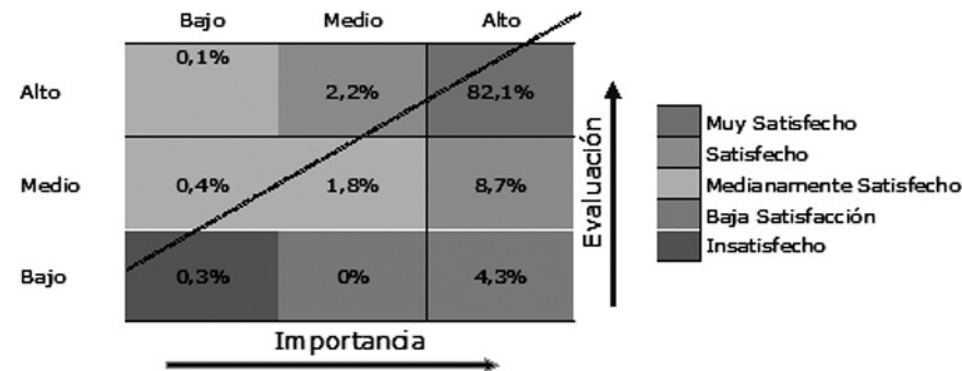

Figura $N^{\circ}$ 6: Matriz Importancia -Evaluación/Resultados Nivel de Satisfacción Espacio Público

En general, el nivel de importancia se valora con un promedio 6,54, mientras que los resultados alcanzados por el aporte del $\mathrm{PQMB}$ se valoran en promedio en un 5,95 . Figura $\mathrm{N}^{\circ} 7$.

Figura $N^{\circ} 7$ : Gráfico importancia y evaluación por Gestión Espacio Público.

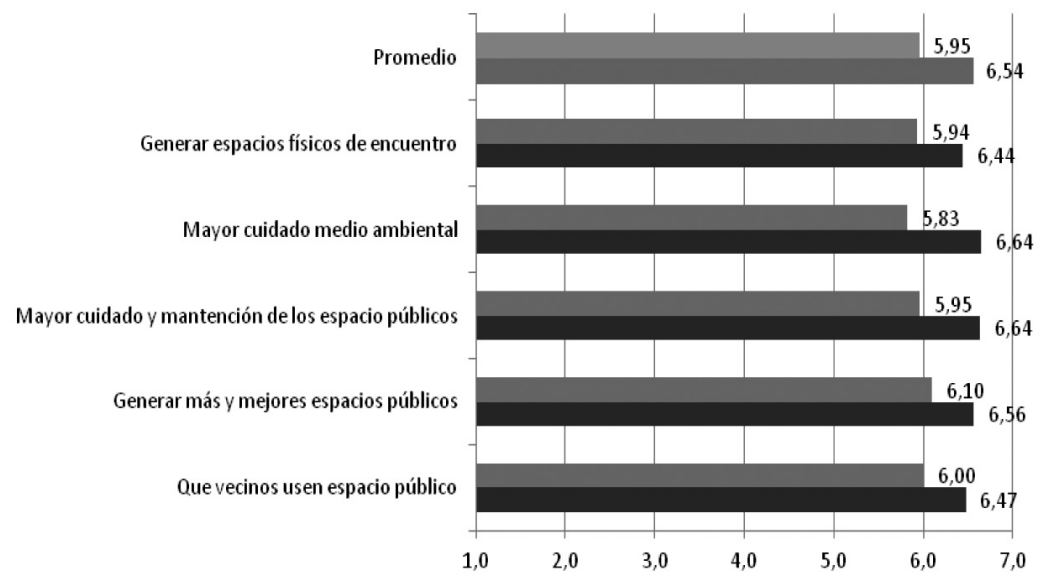

aporte PRB Importancia

La acción más importante para los vecinos es "mayor cuidado medioambiental", junto con "mayor cuidado y mantención del espacio público", mientras que la de menor importancia es "generar espacios físicos de encuentro". El aporte del PQMB mejor evaluado corresponde a "generar más y mejores espacios 
públicos"; mientras que el aporte con menor evaluación corresponde a "mayor cuidado medioambiental".

\section{Nivel de satisfacción Plan de Gestión Social}

Los resultados dan cuenta de que el nivel de importancia de las acciones en el espacio social tiene una mayor valoración que la evaluación del aporte realizado por el PQMB en este ámbito. Figura $\mathrm{N}^{\circ} 8$.

Figura $N^{\circ}$ 8: Matriz importancia-evaluación / resultados nivel de satisfacción Gestión Social.

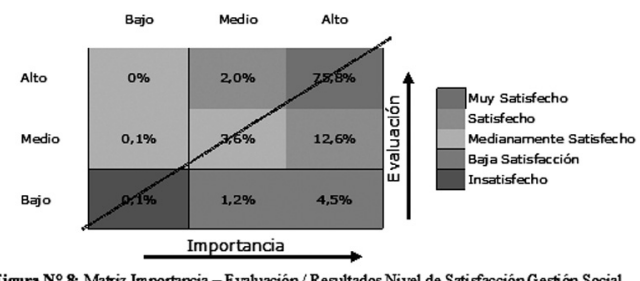

Figura N9: Importancia y evaluación por Gestión Social.

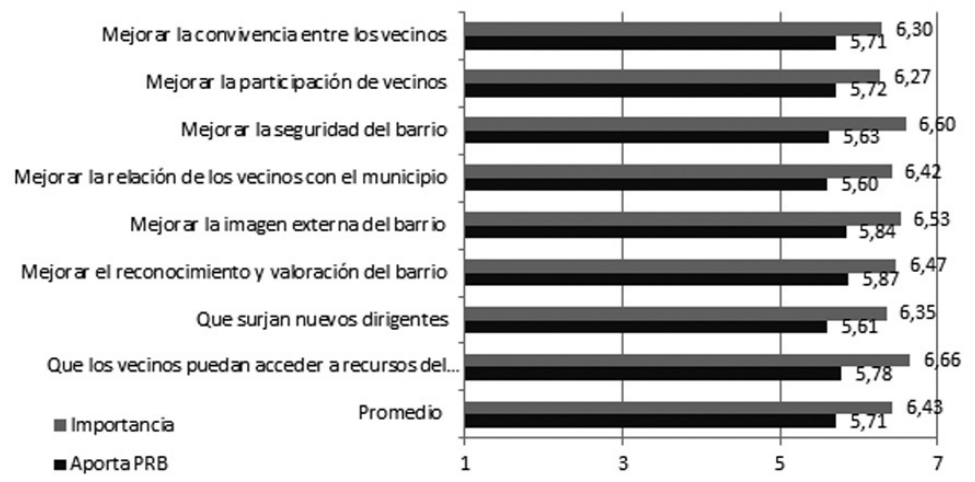

En general, el nivel de importancia de la Gestión Social se valora con un promedio de 6,43, mientras que la evaluación por el aporte del PQMB para materializarlo presenta un promedio 5,71. Figura $\mathrm{N}^{\circ} 9$. 
Lo más importante para los vecinos y vecinas encuestados es "que los vecinos puedan acceder a recursos de otros programas del Estado", la más baja importancia corresponde a "mejorar la participación de los vecinos".

El aporte del PQMB mejor evaluado es "mejorar el reconocimiento y valoración del barrio por parte de los vecinos"; con menor promedio, "mejorar las relaciones entre los vecinos y el municipio". El ítem que presenta mayor diferencia entre valoración del nivel de importancia y la evaluación de los resultados logrados por el PQMB es "mejorar la seguridad del barrio".

\section{Análisis factorial}

Indagamos en la posible relación empírica entre la caracterización que los vecinos hacen de su barrio (preguntas 15 a 18, con un total de 26 ítemes), con el nivel de satisfacción general. A este conjunto de ítemes se aplicó análisis factorial de componentes principales con el propósito de encontrar dimensiones que pudieran estar asociadas al nivel de satisfacción.

La tabla $\mathrm{N}^{\circ} 4$ da cuenta del procesamiento realizado; las comunalidades de cada ítem dan cuenta del nivel de variabilidad que participa del modelo. 
Tabla $N^{\circ}$ 4: Comunalidades.

\section{Comunalidades}

\begin{tabular}{|l|r|r|}
\hline & Inicial & Extracción \\
\hline Barrio Seguro & 1,000 &, 687 \\
Barrio Tranquilo & 1,000 &, 716 \\
Barrio Bonito & 1,000 &, 601 \\
Barrio Amable & 1,000 &, 608 \\
Barrio Solidario & 1,000 &, 577 \\
Barrio Entretenido & 1,000 &, 710 \\
Barrio Limpio & 1,000 &, 610 \\
Barrio Conflictivo & 1,000 &, 640 \\
Barrio Aislado & 1,000 &, 547 \\
Barrio Violento & 1,000 &, 717 \\
Preocupaciòn Contaminación (del aire, ruidos) & 1,000 &, 538 \\
Preocupaciòn Plaga (roedores, termitas) & 1,000 &, 561 \\
Preocupaciòn Mascotas que molestan a vecinos & 1,000 &, 500 \\
Preocupación Microbasurales y/o vertederos clandestinos & 1,000 &, 612 \\
Preocupaciòn Sitios Eriazos inseguros & 1,000 &, 587 \\
Evaluación Veredas & 1,000 &, 468 \\
Evaluación Plazas & 1,000 &, 655 \\
Evaluación Paraderos locomoción & 1,000 &, 448 \\
Evaluación Multicanchas & 1,000 &, 688 \\
Evaluación Sede Social & 1,000 &, 555 \\
Ev Mobiliario sede comunitaria & 1,000 &, 607 \\
Ev Mobiliario plazas y/o parques & 1,000 &, 674 \\
Ev Mobiliario espacios deportivos & 1,000 &, 696 \\
Ev Contenedores de basura & 1,000 &, 461 \\
Ev Colectores de aguas lluvias & 1,000 &, 691 \\
Ev Equi para iluminación de calles, pasajes, veredas & 1,000 &, 652 \\
\hline
\end{tabular}

Método de extracción: Análisis de Componentes principales.

La tabla N5 informa del número de factores extraídos con el método Varimax. El conjunto de indicadores se puede agrupar en seis dimensiones o factores, los que explican el 60,8\% de la varianza. Estos factores se muestran en la tabla $\mathrm{N}^{\mathrm{o}}$ 6; la elipse indica el peso de cada uno de ellos, siendo el de mayor valor el componente $\mathrm{n}^{\circ} 1$ y el de menor valor el componente $\mathrm{n}^{\circ} 6$. 
Tabla $N^{\circ}$ 5: Varianza total explicada en las variables de caracterización de barrio.

\begin{tabular}{|c|c|c|c|c|c|c|c|c|c|}
\hline \multirow[b]{3}{*}{ Componente } & \multicolumn{9}{|c|}{ Varianza total explicada } \\
\hline & \multicolumn{3}{|c|}{ Autovalores iniciales } & \multicolumn{3}{|c|}{$\begin{array}{c}\text { Sumas de las saturaciones al cuadrado } \\
\text { de la extracción }\end{array}$} & \multicolumn{3}{|c|}{$\begin{array}{c}\text { Suma de las saturaciones al cuadrado } \\
\text { de la rotación }\end{array}$} \\
\hline & Total & $\begin{array}{c}\% \text { de la } \\
\text { varianza }\end{array}$ & $\%$ acumulado & Total & $\begin{array}{c}\% \text { de la } \\
\text { varianza }\end{array}$ & $\%$ acumulado & Total & $\begin{array}{c}\% \text { de la } \\
\text { variamea }\end{array}$ & $\%$ acumulado \\
\hline 1 & 6.283 & 24,166 & 24,166 & 6,283 & 24,166 & 24,166 & 4,296 & 16,523 & 16,523 \\
\hline 2 & 3,143 & 12,089 & 36,255 & 3,143 & 12,089 & 36,255 & 2,770 & 10,653 & 27,176 \\
\hline 3 & 2,435 & 9,367 & 45,622 & 2,435 & 9,367 & 45,622 & 2,748 & 10,571 & 37,746 \\
\hline 4 & 1.685 & 6.479 & 52,101 & 1,685 & 6.479 & 52,101 & 2,372 & 9,125 & 46,871 \\
\hline 5 & 1,178 & 4,531 & 56,632 & 1,178 & 4,531 & 56,632 & 2,069 & 7,960 & 54,830 \\
\hline 6 & 1,082 & 4,160 & 60,793 & 1,082 & 4,160 & 60,793 & 1,550 & 5,963 & 60,793 \\
\hline 7 & ,992 & 3,814 & 64,607 & & & & & & \\
\hline 8 & 906 & 3,485 & 68,092 & & & & & & \\
\hline 9 & 877 & 3,373 & 71,465 & & & & & & \\
\hline 10 &, 749 & 2,880 & 74,345 & & & & & & \\
\hline 11 & ,708 & 2,724 & 77,070 & & & & & & \\
\hline 12 & 660 & 2,538 & 79,607 & & & & & & \\
\hline 13 & 646 & 2,483 & 82,090 & & & & & & \\
\hline 14 & ,586 & 2,252 & 84,342 & & & & & & \\
\hline 15 & ,546 & 2,099 & 86,442 & & & & & & \\
\hline 16 &, 503 & 1,936 & 88,378 & & & & & & \\
\hline 17 & 461 & 1,773 & 90,151 & \multirow{8}{*}{\multicolumn{6}{|c|}{$\begin{array}{l}\text { Se indican los componentes de mayor } \\
\text { peso en la varianza son el } 1 \text { y } 2 \text {. Todos } \\
\text { los indicadores evaluados se agrupan } \\
\text { en } 6 \text { dimensiones o factores que } \\
\text { explican el } 60,79 \% \text { de la varianza. }\end{array}$}} \\
\hline 18 & .429 & 1,649 & 91,800 & & & & & & \\
\hline 19 & ,404 & 1,553 & 93,353 & & & & & & \\
\hline 20 & ,359 & 1,380 & 94,733 & & & & & & \\
\hline 21 & ,303 & 1,165 & 95,898 & & & & & & \\
\hline 22 & ,282 & 1,086 & 96,983 & & & & & & \\
\hline 23 & 241 & 928 & 97,911 & & & & & & \\
\hline 24 & ,218 & 837 & 98,748 & & & & & & \\
\hline 25 & 176 & ,676 & 99,424 & & & & & & \\
\hline 26 & 150 & 576 & 100,000 & & & & & & \\
\hline
\end{tabular}

Tabla $\mathbf{N}^{\circ}$ 6: Factores o dimensiones.

\begin{tabular}{|c|c|c|c|c|c|c|}
\hline \multicolumn{7}{|c|}{ Matriz de componentes rotados } \\
\hline & \multicolumn{6}{|c|}{ Componente } \\
\hline & 1 & 2 & 3 & 4 & 5 & 6 \\
\hline Barrio Entretenido & 819 & & & & & \\
\hline Barrio Seguro &, 780 & & & & & \\
\hline Barrio Tranquilo &, 756 & & & & & \\
\hline Barrio Bonito &, 717 & & & & & \\
\hline Barrio Solidario &, 712 & & & & & \\
\hline Barrio Limpio &, 703 & & & & & \\
\hline Barrio Amable &, 700 & & & & & \\
\hline Evaluación Multicanchas & &, 795 & & & & \\
\hline Ev Mobiliario espacios deportivos & & 786 & & & & \\
\hline Ev Contenedores de basura & &, 550 & & & & \\
\hline Evaluación Paraderos locomoción & &, 511 & & & & \\
\hline Evaluación Sede Social & & 499 & & & & \\
\hline Preocupación Microbasurales y/o vertederos clandestinos & & &, 735 & & & \\
\hline Preocupaciòn Plaga (roedores, termitas) & & &, 729 & & & \\
\hline Preocupaciòn Sitios Eriazos inseguros & & &, 716 & & & \\
\hline Preocupaciòn Contaminación (del Aire, ruidos) & & & 695 & & & \\
\hline Preocupaciòn Mascotas que molestan a vecinos & & & 631 & & & \\
\hline Ev Mobiliario plazas y/o parques & & & & ,759 & & \\
\hline Ev Mobiliario sede comunitaria & & & &, 721 & & \\
\hline Evaluación Plazas & & & &, 628 & & \\
\hline Evaluación Veredas & & & &, 592 & & \\
\hline Barrio Violento & & & & & 811 & \\
\hline Barrio Conflictivo & & & & & 769 & \\
\hline Barrio Aislado & & & & & .720 & \\
\hline Ev Colectores de aguas Iluvias & & & & & &, 793 \\
\hline Ev Equi para iluminación de calles, pasajes, veredas & & & & & & 652 \\
\hline
\end{tabular}

El análisis factorial arroja como resultado (tabla $\mathrm{N}^{\circ}$ 6) la agrupación de los indicadores evaluados en seis dimensiones o ámbitos. La interpretación de cada uno nos lleva a etiquetarlos como: 
- Componente 1: Convivencia.

- Componente 2: Equipamiento.

- Componente 3: Medio ambiente e higiene.

- Componente 4: Espacio público.

- Componente 5: Conflictividad e inseguridad.

- Componente 6: Servicios.

El análisis factorial de componentes principales indica que los encuestados y encuestadas, al evaluar las características del barrio, otorgan mayor significado e importancia al componente 1, luego al componente 2 y así sucesivamente. En la práctica, esto significa que los vecinos y vecinas encuestados evalúan como de primera importancia y significación los aspectos asociados a la convivencia y habitabilidad del barrio. En segundo lugar, los equipamientos del barrio.

La tercera dimensión está referida a la problemática ambiental, que incide negativamente en la percepción del barrio amable y habitable. Como cuarta prioridad, al espacio público en el cual se encuentran por recreación o asociación los vecinos y vecinas del barrio. Una quinta prioridad en la evaluación corresponde a temas asociados con la conflictividad, violencia y aislamiento. En sexto lugar están dos servicios públicos: evacuación colectores de aguas lluvias y alumbrado público.

Figura $\mathrm{N}^{\circ} 10$ : Gráfico promedio según ámbitos de evaluación de las características del barrio.

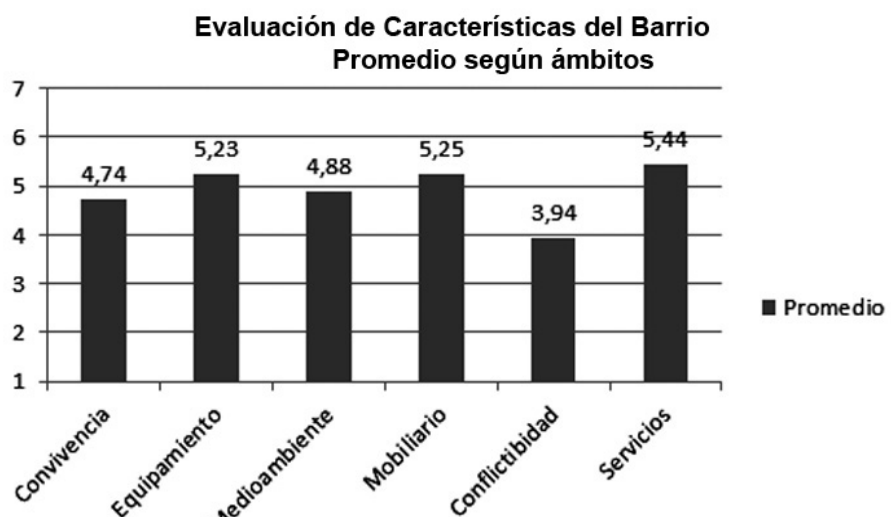


Considerando esta configuración de factores, ámbitos o dimensiones, se procedió a promediar los ítemes que conforman cada factor, a objeto de conocer la evaluación de cada factor o dimensión. El promedio por dimensión se presenta en la figura $\mathrm{N}^{\circ} 10$.

Tabla $N^{\circ}$ 7: Prueba $\mathrm{T}$ para muestras independientes $95 \%$ nivel de confianza factores con diferencias de medias estadísticamente significativas para nivel de satisfacción ${ }^{5}$.

\begin{tabular}{|c|c|c|c|c|}
\hline Factores & $\begin{array}{l}\text { Categorías según pro- } \\
\text { medio de factor }\end{array}$ & $\begin{array}{l}\mathrm{N} \text { de } \\
\text { casos }\end{array}$ & $\begin{array}{l}\text { Promedio } \\
\text { Satisfacción }\end{array}$ & $\begin{array}{l}\text { Significación } \\
\text { dif. de media } \\
\text { Satisfacción }\end{array}$ \\
\hline \multirow{2}{*}{$\begin{array}{l}\text { Factor } 1 \\
\text { Conviven- } \\
\text { cia }\end{array}$} & Bajo la media $<4,7$ & 361 & 5,69 & \multirow{2}{*}{0,000} \\
\hline & Media o mayor $>=4,7$ & 514 & 6,12 & \\
\hline \multirow{2}{*}{$\begin{array}{l}\text { Factor } 2 \\
\text { Equipa- } \\
\text { miento }\end{array}$} & Bajo la media < 5,2 & 364 & 5,61 & \multirow{2}{*}{0,000} \\
\hline & Media o mayor $>=5,2$ & 508 & 6,18 & \\
\hline \multirow{2}{*}{$\begin{array}{l}\text { Factor } 3 \\
\text { Medio } \\
\text { Ambiente }\end{array}$} & Bajo la media $<4,8$ & 356 & 5,83 & \multirow[b]{2}{*}{0,001} \\
\hline & Media o mayor $>=4,8$ & 518 & 6,03 & \\
\hline \multirow{2}{*}{$\begin{array}{l}\text { Factor } 4 \\
\text { Mobiliario }\end{array}$} & Bajo la media $<5,2$ & 360 & 5,67 & \multirow{2}{*}{0,000} \\
\hline & Media o mayor $>=5,2$ & 515 & 6,14 & \\
\hline \multirow{2}{*}{$\begin{array}{l}\text { Factor } 5 \\
\text { Conflicti- } \\
\text { vidad }\end{array}$} & Bajo la media $<0,115$ & & & \multirow[b]{2}{*}{0,993} \\
\hline & $\begin{array}{l}\text { Media o mayor }>= \\
0,1142\end{array}$ & 876 & & \\
\hline \multirow{2}{*}{$\begin{array}{l}\text { Factor } 6 \\
\text { Servicios }\end{array}$} & Bajo la media < 5,4 & 323 & 5,78 & \multirow{2}{*}{0,000} \\
\hline & Media o mayor $>=5,4$ & 537 & 6,06 & \\
\hline
\end{tabular}

Con el fin de identificar la existencia de variables que podrían incidir en la evaluación del nivel de satisfacción de los vecinos, se procedió a testear la diferencia de medias, a través de la aplicación de la Prueba T.

El resultado obtenido permite interpretar que la variable "nivel de satisfacción", está asociada a cinco de los seis factores, según queda establecido por las diferencias estadísticamente signi-

Se indagaron variables demográficas, sexo, años viviendo en el barrio, no encontrándose diferencias significativas respecto del nivel de satisfacción. 
ficativas $(\mathrm{p}=<0,05)$ entre las distintas categorías de las variables especificadas en la tabla $N^{\circ} 7$. Se puede observar que el factor cinco, "conflictividad", no discrimina respecto de la evaluación de la satisfacción.

\section{Resultados y discusión}

Esta investigación ha permitido comprobar que el Modelo de Análisis de Importancia-Desempeño es de utilidad para la medición del nivel de satisfacción de los vecinos y vecinas respecto de la intervención del PQMB, así como también aportar evidencia de algunos factores asociados a la valoración que los vecinos y vecinas de los barrios tienen respecto del nivel de satisfacción.

Según lo indicado por Kitcharoen (2004, citado en Gelvez, 2010), al utilizar el modelo de Análisis de Importancia-Desempeño es posible identificar desde la perspectiva del cliente, en este caso de los habitantes de los barrios, los aspectos en que el PQMB presentó mayores y menores desempeños respecto de la evaluación de los resultados, así como respecto de cuáles eran los aspectos de mayor relevancia.

Los resultados de la evaluación Análisis de Importancia-Desempeño, de acuerdo con lo sostenido por Santomá (2008, citado en Gelvez, 2010), permiten relevar las expectativas y necesidades de los habitantes de los barrios y que, de acuerdo con los resultados, serían los siguientes:

1. Desde una perspectiva general, a pesar del tiempo trascurrido desde el cierre de la intervención del PQMB (dos años), todos los barrios presentaron un alto nivel de satisfacción por la intervención realizada.

2. El modelo permite evaluar los resultados del Programa de Recuperación de Barrios, identificando que, si bien existe un nivel de satisfacción alto, en la mayoría de las intervenciones los vecinos evalúan por debajo de sus expectativas. 
3. La diferencia de promedio entre la dimensión evaluación e importancia de 1,07 nos indica que, aunque la satisfacción es alta, existe una brecha entre las expectativas de los vecinos y vecinas, ya que estas son mayores a la evaluación de la intervención del PQMB. Los vecinos y vecinas esperaban más del PQMB.

4. Los resultados del nivel de satisfacción del Plan de Gestión de Obras nos muestran que las obras de mayor relevancia para los vecinos son las complementarias en condominios sociales, seguidas del alumbrado. Ambos tipos de obras están asociadas a una mayor cercanía, el espacio más cotidiano y próximo del barrio.

5. Los resultados del nivel de satisfacción de la Gestión del Espacio Público indican que el ítem más importante para los vecinos es "mayor cuidado medioambiental", pero a la vez es la gestión con más baja evaluación en cuanto a los resultados logrados por el PQMB. Aquello indicaría que es necesario desarrollar a futuro mayores acciones y recursos que permitan mejores resultados en esta materia.

6. Los resultados del nivel de satisfacción de la Gestión del Espacio Social indican que la acción más significativa para los encuestados y encuestadas es "que los vecinos puedan acceder a recursos de otros programas del Estado", mientras que el resultado del PQMB con más baja evaluación es "mejorar las relaciones entre los vecinos y el municipio".

7. El análisis de componentes principales nos da indicios respecto de la importancia que los encuestados y encuestadas otorgan al factor Convivencia, lo que nos indica la percepción del barrio como un espacio social, desde el cual es percibido positivamente. Los factores equipamiento, medio ambiente e higiene, y espacio público, responden a una condición de habitabilidad del espacio residencial.

8. El análisis de componentes principales entrega evidencia de que la característica de conflictividad del barrio no discrimina en la construcción de la evaluación de la satisfacción. 
Finalmente, y de acuerdo a lo sostenido por Forero y Ospina (2013), se podría afirmar que el alto grado de satisfacción de los vecinos con los resultados del $P Q M B$ se debe a que los aspectos intervenidos, si bien presentan una evaluación inferior al grado de importancia que los vecinos les asignan, son todos aspectos sensibles y de relevancia de las necesidades de los habitantes de los barrios.

\section{Conclusiones}

El creciente déficit urbano que emerge de poblaciones construidas y conformadas por las diferentes etapas de las políticas habitacionales, pone de manifiesto la necesidad de generar políticas públicas que den cobertura a la habilitación, recuperación y regeneración de una gran extensión de la ciudad, una de estas es el Programa Quiero Mi Barrio.

Es necesario generar conocimiento respecto de cómo una política pública, que se plantea participativa, incide en la percepción de la satisfacción de los habitantes con su entorno residencial a partir de las intervenciones que se plantean, con el objeto de identificar aquellos aspectos en los que es valorada su acción por parte de los vecinos y vecinas que han sido sujetos del proceso.

La investigación realizada permite verificar que los conceptos de "evaluación" y de "importancia" de la intervención efectuada por el Programa Quiero Mi Barrio, evaluadas por los propios vecinos y vecinas, son fuente de información relevante para el mejoramiento de los servicios y productos ejecutados por este Programa en futuros barrios. Estos aportan en la identificación de los aspectos de mayor relevancia para los habitantes, los que, al tenerlos en consideración, podrían mejorar la toma de decisiones que aporten a la calidad y oportunidad en la entrega de servicios.

La utilización y análisis de la matriz de importancia y evaluación como variables del constructo "satisfacción", permitieron 
identificar aquellos indicadores o atributos de servicios y productos que deben corregirse.

\section{Referencias}

Andreu, L. y Bigné J.E. (2004). Modelo cognitivo-afectivo de la satisfacción en servicios de ocio y turismo. Cuadernos de Economía y Dirección de la Empresa, 21, 89-120.

Blanco, E. (2007). Evaluación de la calidad del servicio del Centro Comercial Las Antillas. Trabajo de Diploma en opción al Título de Lic. en Economía. Cuba: Centro Universitario de Las Tunas, Departamento Docente de Economía.

Breymann, H. (2015). Identidad, apropiación, sentido de comunidad y satisfacción residencial: claves de análisis para los estudios urbanos y la planificación. El caso del barrio Embajadores, Madrid. Tesis (Doctoral). E.T.S. Arquitectura (UPM).

Feijoo, R., Caro, A., Gil, M.T. \& Quintana, D. (2002). Satisfacción del consumidor: modelos explicativos. España: Universidad de Las Palmas de Gran Canaria.

Femenía, O. (s. f.). La imagen de un destino turístico como herramienta del marketing. Recuperado el 9 de mayo de 2016 de http:// www.eumed.net/libros- gratis/2011c/986/indice.htm

Forero, A. y Ospina D. (2013). El diseño de experiencias. [Experience design]. Revista de Arquitectura, 15, 78-83.

Gelvez, J. (2010). Estado del arte de modelos de medición de la satisfaccion del cliente. Trabajo de grado para optar el título de Ingeniera Industrial. Recuperado en agosto de 2014, de Universidad Industrial de Santander. Facultad de Ingenierías Fisicomecánicas. Escuela de Estudios Industriales y Empresariales. Bucaramanga: http://repositorio.uis.edu. co/jspui/bitstream/123456789/5293/2/136447.pdf

MINVU (2008). Programa Quiero Mi Barrio. Santiago de Chile. Recuperado en mayo de 2016 de http://pdba.georgetown.edu/Security/citizensecurity/chile/politicas/QuieromiBarrio.pdf

MINVU (2009). Encuesta de caracterización familiar y percepción del PRB: Un análisis de los vecinos, sus comunidades y sus barrios. Cuadernos de Barrio $\mathrm{N}^{\circ}$ 1. Santiago de Chile. Recuperado en mayo de 2016 de: http:/ / www.minvu.cl/opensite_20141028132429.aspx 
MINVU (2010). Resultados Encuesta Diagnóstico y Satisfacción Programa de Recuperación de Barrios. Etapa del Piloto. Recuperado en mayo de 2016 de: http://www.minvu.cl/opensite_20141028132429.aspx

MINVU (2013). Documentos de Trabajo Fase I y Fase II, Programa Recuperación de Barrios. Intervención en Barrios Vulnerables. Santiago de Chile, documento sin publicar.

Oliver, R. (1989): Processing of the satisfaction response in consumption: a suggested framework and research propositions. Journal of Consumer Satisfaction, Dissatisfaction and Complaining Behavior, 2, 1-16. 